\title{
Effect of methyl jasmonate on production of 20- hydroxyecdysone and turkesterone in hairy roots of Silene linicola C.C.Gmelin
}

\author{
Anna A. Erst ${ }^{1, *}$, Larisa N. Zibareva ${ }^{2}$, and Elena S. Filonenko ${ }^{2}$ \\ ${ }^{1}$ Central Siberian botanical garden of SB RAS Zolotodolinskaya st., 101, Novosibirsk, 630090, \\ Russia \\ ${ }^{2}$ National research Tomsk state university, Lenin avenue, 36, Tomsk, 634050, Russia
}

\begin{abstract}
Methyl jasmonate (MeJ) affects the regulation of secondary metabolism, and it is considered to be a promising elicitor in the culture of cells, tissues and plant organs. High-performance liquid chromatography method was used to identify the composition of ecdysteroids in hairy roots of Silene linicola. MeJ was found to have a stimulating effect on ecdysteroid biosynthesis in this culture. Addition of MeJ at a concentration of $100 \mu \mathrm{M}$ increased the biosynthesis of 20-hydroxyecdysone by $74 \%$ (day 3 ), and that of turkesterone by $35 \%$ (day 6 ). The share of turkesterone in total ecdysteroid content in the investigated samples was up to $60 \%$, and the content of 20-hydroxyecdysone was up to $30 \%$. The study shows that MeJ is a promising stimulator of ecdysteroid biosynthesis in hairy roots of S. linicola.
\end{abstract}

\section{Introduction}

In recent years, a great deal of attention has been paid to the family Caryophyllaceae Juss. as a new source of ecdysteroids due to the discovery of this class of compounds in its composition. The representatives of this family are characterized by diverse composition of ecdysteroids, the presence of many new compounds not found in any other species, and high content of major components of total ecdysteroids $[1,2]$. These species are promising biotechnological objects. The most common ecdysteroids in Caryophyllaceae Juss. are 20hydroxyecdysone (20E), polypodine B, 2-deoxy-20-hydroxyecdysone, ecdysone, viticosterone $\mathrm{E}$, and integristerone $\mathrm{A}$.

A number of studies aim to develop new methods for increasing the biosynthetic ability of in vitro cultures. Some scientists suggest not to activate one or more key genes of secondary metabolism, but to develop approaches to remove inhibitor blocks and to shift the regulatory balance towards activation of the entire biosynthetic pathways [3]. Other scientists investigate the processes of enhanced secondary metabolism of in vitro cultures using proteomic methods that enable regulation of enzymes involved in biosynthesis of secondary metabolites [4]. However, most of the studies on regulation of in vitro bioactive compound accumulation focus on optimization of media and culture conditions, and on

\footnotetext{
* Corresponding author: annaerst@yandex.ru
} 
selection of growth regulators, elicitors and precursors of secondary metabolite synthesis $[5,6]$. Methyl jasmonate $(\mathrm{MeJ})$ is one of the elicitors widely used in the culture of cells, tissues and plant organs [7-10]. It is shown that it has a stimulating effect on the content of ecdysteroids, in particular 20E, in plants in vitro and ex vitro $[11,12]$.

The current study aims to develop a strategy for elicitor-stimulated biosynthesis of phytoecdysteroids in hairy roots of Silene linicola, in particular 20E and turkesterone.

\section{Materials and methods}

\subsection{Elicitation of hairy root cultures}

Hairy roots of $S$. linicola were cultured on liquid $\mathrm{B}_{5}$ medium [13] with the addition of 500 $\mathrm{mg} \cdot \mathrm{l}^{-1}$ casein hydrolysate at 30-42 day interval $[14,15]$. Elicitation of ecdysteroid accumulation was carried out by adding MeJ to media at the 9th passage at the end of subculturing (day 30) at a concentration of 100 or $200 \mu \mathrm{M}(22.43$ or $44.86 \mathrm{mg}$ of MeJ was dissolved in $4 \mathrm{ml}$ of $70 \%$ ethanol, respectively) together with $30 \mathrm{ml}$ of $\mathrm{B}_{5}$ medium (ethanol concentration of $0.28 \%$ ). To assess the effect of $\mathrm{MeJ}$ on growth characteristics of culturing and ecdysteroid biosynthesis, some of the samples were cultured only with the addition of ethanol solvent (at a concentration similar to that in the experiment with MeJ) and $30 \mathrm{ml}$ of B5 medium. The samples were analyzed after 3, 6 and 12 days, after the components were added to media (on culture day 33, 36 and 42, respectively). Two controls were used in the study. Control 1 (C1) was the sample of hairy roots at the beginning of the experiment (culture day 30), control 2 (C2) was the sample at the end of the experiment (culture day 42), with no MeJ added.

\subsection{Methods of research of biologically active substances of hairy root cultures}

Extracts from 1-2 g of hairy root cultures were produced through repercolation with $70 \%$ ethanol solution followed by concentration to $2 \mathrm{ml}$ with IKA rotary evaporator HB 10 digital (Germany). The analysis of phytoecdysteroids was performed in the Laboratory of Phytochemistry, SBG TSU (Tomsk, Russia) using high performance liquid chromatography according to the procedure described in $[14,15]$.

\section{Results}

\subsection{Identification of ecdysteroids in ethanol extracts of hairy root cultures by HPLC}

Turkesterone, 20E and polypodin B were identified in ethanol extracts of hairy root cultures by comparing the retention times of standards and peaks of compounds with absorption maxima characteristic of ecdysteroids at $\lambda 240-250 \mathrm{~nm}$. 20E was found in all the samples, turkesterone was found in virtually all samples except for sample 200-12, whereas polypodin B was detected only in control sample C2 on culture day 42. HPLC analysis of the investigated samples of hairy roots of S. linicola showed that a number of ecdysteroids, including nonpolar ones, are synthesized in the retention time interval of 23.7-51.0 min under the preditermined experimental conditions. 


\subsection{Quantitative determination of ecdysteroids in extracts of hairy root cultures}

The results of the quantitative analysis of the ecdysteroid content in the samples in terms of the equivalent amount of absolutely dry raw materials are provided in the table.

Table. Ecdysteroid content in extracts of hairy roots of S. linicola.

\begin{tabular}{|c|c|c|c|c|}
\hline No. & Samples & $\begin{array}{c}\text { 20E content, \% } \\
(\mathrm{x} \pm \mathrm{m})\end{array}$ & $\begin{array}{c}\text { Turkesterone } \\
\text { content, \% } \\
(\mathrm{x} \pm \mathrm{m})\end{array}$ & $\begin{array}{c}\text { Total ecdysteroid } \\
\text { content, \% }\end{array}$ \\
\hline 1 & $\mathrm{C} 1$ & $0.023 \pm 0.0008$ & $0.016 \pm 0.0003$ & $0.063 \pm 0.0006$ \\
\hline 2 & $\mathrm{C} 2$ & $0.014 \pm 0.0004$ & $0.012 \pm 0.0005$ & $0.055 \pm 0.0005$ \\
\hline 3 & $0-3$ & $0.019 \pm 0.0002$ & $0.045 \pm 0.0013$ & $0.100 \pm 0.0008$ \\
\hline 4 & $0-6$ & $0.018 \pm 0.0005$ & $0.054 \pm 0.0009$ & $0.094 \pm 0.0007$ \\
\hline 5 & $0-12$ & $0.016 \pm 0.0003$ & $0.028 \pm 0.0012$ & $0.073 \pm 0.0008$ \\
\hline 6 & $100-3$ & $0.033 \pm 0.0009$ & $0.038 \pm 0.0037$ & $0.107 \pm 0.0023$ \\
\hline 7 & $100-6$ & $0.030 \pm 0.0011$ & $0.073 \pm 0.0023$ & $0.138 \pm 0.0017$ \\
\hline 8 & $100-12$ & $0.011 \pm 0.0003$ & $0.046 \pm 0.0007$ & $0.134 \pm 0.0005$ \\
\hline 9 & $200-3$ & $0.025 \pm 0.0012$ & $0.010 \pm 0.0003$ & $0.056 \pm 0.0008$ \\
\hline 10 & $200-6$ & $0.018 \pm 0.0007$ & $0.023 \pm 0.0014$ & $0.061 \pm 0.0011$ \\
\hline 11 & $200-12$ & $0.021 \pm 0.0003$ & - & $0.038 \pm 0.0003$ \\
\hline
\end{tabular}

Note: $\mathrm{C} 1$ - control, start of the experiment; $\mathrm{C} 2$ - control, end of the experiment (day 12); 03 - ethanol, day 3; 0-6 - ethanol, day 6; 0-12 - ethanol, day 12; 100-3 - $100 \mu \mathrm{M} \mathrm{MeJ}$, day 3; 100-6 - $100 \mu \mathrm{M}$ MeJ, day 6; 100-12 - $100 \mu \mathrm{M}$ MeJ, day 12; 200-3 - $200 \mu \mathrm{M} \mathrm{MeJ,} \mathrm{day}$ 3; 200-6 - $200 \mu \mathrm{M}$ MeJ, day 6; 200-12 - $200 \mu \mathrm{M} \mathrm{MeJ,} \mathrm{day} 12$.

As can be seen from the table, the content of $20 \mathrm{E}$ in the control decreased from 0.023 to $0.014 \%$. The content of $20 \mathrm{E}$ in the samples with no elicitor, but with ethanol added, was observed to decrease as well. However, this led to the active synthesis of turkesterone, which attained its maximum on day 6 .

The addition of $100 \mu \mathrm{M} \mathrm{MeJ}$ results in an increased content of $20 \mathrm{E}$ on day 3 as compared to that in the control and samples cultured with ethanol. The pattern of changes in turkesterone after the addition of $100 \mu \mathrm{M}$ MeJ is similar to that in the samples with ethanol. The maximum concentration of ethanol and total ecdysteroid content (table) can be observed on culture day 6 . However, the comparison of turkesterone concentrations showed that its content in sample 100-6 exceeds that in the control by a factor of $4.5-6$, and in sample $0-6$ - by a factor of 1.4. In control samples, turkesterone amounts to $25 \%$ of total ecdysteroid content, whereas in samples with ethanol and those with $100 \mu \mathrm{M} \mathrm{MeJ}$, it attains $40-60 \%$ and $35-52 \%$, respectively. At the same time, the content of $20 \mathrm{E}$ in total ecdysteroids varies from 8 to $30 \%$.

Weak inhibition of ecdysteroid synthesis can be observed in the experiment with MeJ added at a concentration of $200 \mu \mathrm{M}$ (table).

\section{Discussion}

MeJ is a widely used elicitor in the culture of cells, tissues and plant organs in vitro. Thus, the addition of MeJ caused a 2-3-fold increase in the content of $20 \mathrm{E}$ in suspension and hairy root cultures of Ajuga turkestanica (Regel) Briq. [11]. A positive effect of MeJ on the accumulation of phytoecdysteroids was also observed for intact plants of Spinacia oleracea 
L. [12]. The results of our studies revealed a stimulating effect of MeJ on ecdysteroid biosynthesis in hairy roots of S. linicola. It should be noted that the use of sodium acetate (the precursor of ecdysteroid biosynthesis) resulted in a different composition of ecdysteroids in hairy roots of S. linicola [14]; in particular, a valuable and rare turkesterone was not identified. However, ecdysone and 2-deoxy-20-hydroxyecdysone and 2-deoxy ecdysone typical of Caryophyllaceae Juss. were found in S. linicola.

The ecdysteroid composition in intact plants of S. linicola was found to include 20E, polypodine $\mathrm{B}$, ecdysone, 2-deoxy-20-hydroxyecdysone, viticosterone $\mathrm{E}$, turkesterone, and integristerone A [16]. In the introduced species of S. linicola, the major component is $20 \mathrm{E}$, and the 20E to turkesterone ratio is $367: 1$ [16]. The study has shown that when $100 \mu \mathrm{M}$ $\mathrm{MeJ}$ is supplemented, the content of turkesterone in hairy roots of S. linicola exceeds that of $20 \mathrm{E}$ by a factor of $1.7-3$ on day 6 . Hydroxylation of $20 \mathrm{E}$ is likely to occur to the $11 \mathrm{C}$ position, which leads to turkestherone synthesis, or to the $5 \mathrm{C}$ position, which causes polypodine B synthesis, and other positions of the steroid nucleus. At the same time, the content of the initial component (20E) decreases, but the content of other components is observed to increase.

Thus, hairy roots of S. linicola can be the source of a rare ecdysteroid, turkesterone, in the experiment scaling. To date, it has been identified in 5 plant species only: $A$. turkestanic, Vitex glabrata, Vitex canescens (Lamiaceae), Leuzea carthamoides (Asteraceae), S. linicola (Caryophyllaceae), and Tapinella panuoides (Fungi) (http: ecdybase.org/). Addition of $\mathrm{MeJ}$ at a concentration of $100 \mu \mathrm{M}$ is a promising technique for stimulating biosynthesis of such valuable ecdysteroids as $20 \mathrm{E}$ and turkesterone in hairy roots of $S$. linicola.

The work was carried out with financial support of the budgetary project of Central Siberian Botanical Garden, SB RAS Assessment of morphogenetic potential of North Asian plant populations by experimental methods (AAAA-A17-117012610051-5). We also acknowledge the support of the RFBR grant, project № 16-44-7000634 Study of medicinal plants of Tomsk region and adaptive capabilities of introducents in order to create a raw material base of species, which are promising for treatment of socially significant diseases.

The study employed in vitro material from collection No. USU_440534 Collection of living plants indoors and outdoors.

\section{References}

1. L. Zibareva, V. Volodin, Z. Saatov, T. Savchenko, P. Whiting, R. Lafont, L. Dinan, Phytochemistry 64, 499-517 (2003)

2. L. Zibareva, O. Volkova, S. Morozov, E. Chernyak, Phytochemistry 1, 71-75 (2017)

3. V. Bulgakov, T. Avramenko, Biotechnol Lett. 37, 1719-1727 (2015)

4. M. J. Martínez-Esteso, A. Martínez-Márquez, S. Sellés-Marchart, J. A. MoranteCarriel, R. Bru-Martínez, Front Plant Sci. 6, 504 (2015)

5. A. Giri, M. L. Narasu, Biotechnology Advances 18, 1-22 (2000)

6. M. I. Georgiev, J. Weber, A. Maciuk, Appl Microbiol Biotechnol 83, 809-823 (2009)

7. L. Shabania, A. A. Ehsanpoura, G. Asgharib, J. Emamib, Russian Journal of Plant Physiology 56, 621-626 (2009)

8. K. P. Martin, A. Sabovljevic, J. Madassery , J. Crop Sci. Biotech. 14, $205-212$ (2011)

9. M. Ram, K. V. Prasad, S. K. Singh, B. S. Hada, S. Kumar, Plant Cell Tiss. Organ Cult., 113, 459-467 (2013) 
10. B. Chodisetti, K. Rao, S. Gandi, A. Giri, In Vitro Cell.Dev.Biol.-Plant 51, 88-92 (2015)

11. D. M.Cheng, G. G.Yousef, M. H.Grace, R. B.Rogers, J. Gorelick-Feldman, I. Raskin, M. A. Lila, Plant Cell Tiss. Organ. Cult. 93, 73-83 (2008)

12. E. A. Schmelz, R. J.Grebenok, T. E.Ohnmeiss, W. S. Bowers, Chem. Ecol. 26, 28832896 (2000)

13. O. L. Gamborg, D. E. Eveleigh, Can J Biochem. 46, 417-421 (1968)

14. A. A. Erst, T. V. Zheleznichenko, A. A. Badulina, L. N. Zibareva, O. V. Kovzunova, Plant Cell Biotechnology and Molecular Biology 17, 326-334 (2016)

15. A. Erst, L. Zibareva, T. Zheleznichenko, O. Kovzunova, Tomsk State University Journal, Biology 37, 17-30 (2017)

16. L. N. Zibareva, Phytoecdysteroids of plants of the family Caryophyllaceae (Germany LAP Lambert Academic Publishing GmbH\&Co, Saarbrücken, 2012) 